Florina-Mădălina Oniceanu ${ }^{1}$, Ileana Ion ${ }^{1}$, Elena Leu ${ }^{2}$, Adriana Apostol ${ }^{2}$, Cecilia Adumitresi ${ }^{1}$, Ninela Rădulescu ${ }^{1}$, Cristina Farcaş ${ }^{1}$, Anabella-Cristiana Ştefănescu' ${ }^{1}$, R. Chirică ${ }^{1}$

\title{
Rare Immunodeficiency In A Two-Months-Old Infant
}

${ }^{1}$ Department 1 - Preclinic disciplines, Faculty of Medicine, "Ovidius" University

${ }^{2}$ Constanța County Emergency Clinical Hospital

\begin{abstract}
Chronic granulomatous disease is a diverse group of hereditary diseases characterized by neutrophils incapacity or difficulty to form reactive oxygen compounds, most important, the superoxide radical, used to kill certain pathogens. The consequence is recurrent infections with germs with normally low pathogenicity and formation of supurative granulomas. The authors present a pathology case of immunodeficiency rarely seen in pediatrics: chronic granulomatous disease in a 2 months old infant.

The positive diagnosis was based on clinical presentation, dominated by lymphadenopathy, pemphigoid skin lesions, bilateral ear secretion, growth failure, hepatomegaly.

Physical presentation correlated with laboratory studies (Bursttest), chest radiography and CT imaging, moderate inflammatory syndrome, low platelets level and the presence of fibrin monomers suggested a neonatal sepsis having on the background a X-linked immunodeficiency (the patient being the only male survivor in his family, with 4 healthy sisters).

The specific feature in this case was early onset of the disease, with clinical, but most important, pulmonary radiological aspect characteristic for chronic granulomatous disease.

The prognosis was poor; the baby is readmitted in the hospital after 2 months and he dies, having clinical aspect of sepsis unresponsive to the applied intensive therapy. The histological findings enhanced numerous pulmonary and hepatic microabscesses.
\end{abstract}

Key words: chronic granulomatous disease, sepsis, lymphadenoapthy, Bursttest

\section{Introduction}

Chronic granulomatous disease (CGD) is a diverse group of hereditary diseases characterized by disorder of phagocytic cells, with inability to produce bactericidal superoxide anions $\left(\mathrm{O}^{2-}\right)$. This leads to recurrent life threatening bacterial and fungal infections. CGD is known to be caused by a defect in the nicotinamide adenine dinucleotide phosphate (NADPH), reduced form, oxydase enzyme complex of phagocytes. CGD refers to the characteristic granulomas that develop in response to the chronic inflammation [1].

It can either be X-linked or an autosomal recessive inherited disease. Four different genetic types have been identified, among which deficiency of the heavy chain in the cytochrome (X-linked) is the most frequent.

CGD is listed as a "rare disease" by the Office of Rare Diseases (ORD) of the National Institutes of Health (NIH) in USA. This means that CGD affects less than 200,000 people in the US population. CGD occurs once in every one million persons. Orphanet, a consortium of European partners, currently defines a condition rare when it affects 1 person per 2,000, so they list CGD as a "rare disease". There are no statistics for CGD in Romania. CGD is far more common in males than females by a ratio of 4 to $1[1]$.

\section{Material and Methods}

Department 1 - Preclinic disciplines, Faculty of Medicine

145 Tomis Blv.

Tel: +40-722-648-830

E-mail: oniceanuflorinamadalina@yahoo.com
The authors present the case of a 2 months old suckling, male, diagnosed with CGD, based on 
the clinical aspect, imaging findings and confirmed using Bursttest.

\section{Results}

The authors evaluated the clinical aspects, the evolution and treatment in this case of chronic granulomatous disease.

On examination, the 2 months old baby had: - growth failure, with a weight of $4600 \mathrm{~g}$, birth weight $3900 \mathrm{~g}$ (ponderal index 0.85);

- feeding difficulties;

- pale skin, with multiple pemphigoid lesions behind the ear, axillary and inguinal;

- bilateral ear secretion;

- lymphadenopathy in all peripheral lymph nodes, isolated or conglomerated, $2-3 \mathrm{~cm}$ in diameter, without modifying the skin above, very painful when pressed;

- $\quad$ anterior fontanelle normotensive;

- no pulmonary modified sounds, a respiratory rate of 34 respirations/min, an oxygen saturation of 97\% in room air;

- pulse of 130 beats/min, normal heart sounds, no murmurs;
- moderate abdominal distention, with hepatomegaly, prehepatic diameter $11 \mathrm{~cm}$, mild increased consistency, mild umbilical hernia.

A pulmonary radiography revealed nodular bilateral infiltrate with mediastinal adenomegalies.

Hematologic studies revealed:

- WBC of $15,500 / \mathrm{mmc}$, with a differential count of $77 \%$ neutrophils, $15 \%$ lymphocytes, monocytes $6 \%$ and $1 \%$ eosinophils.; hemoglobin 7.3 $\mathrm{g} / \mathrm{dl}$, hematocrit $23.3 \%$, platelet count $132,000 / \mathrm{mmc}$.

- Inflammatory syndrome, with CRP $3.25 \mathrm{mg} /$ $\mathrm{dl}$, but with low fibrinogen $112 \mathrm{mg} / \mathrm{dl}$ and D-dimers present $(0.79 \mu \mathrm{g} / \mathrm{dl})$.

- The infant was negative for RPR, IgM antibodies for Toxoplasma gondi, Ig $G$ Toxocara canis, IgM Epstein Barr.

The culture form the skin lesions revealed Pseudomonas aeruginosa, the culture from the ear secretion revealed also Pseudomonas aeruginosa and Methicillin-resistant Staphylococcus aureus. Blood culture revealed Staphylococcus saprophyticus.

A lombar puncture was performed and the cerebrospinal fluid studies were normal.

Diagnosis algorithm started considering the clinical aspect, dominated by the lymphadenopathies, pemphigoid skin lesions, bilateral ear secretion, growth failure, hepatomegaly and important imaging findings discordant to the clinical aspect.

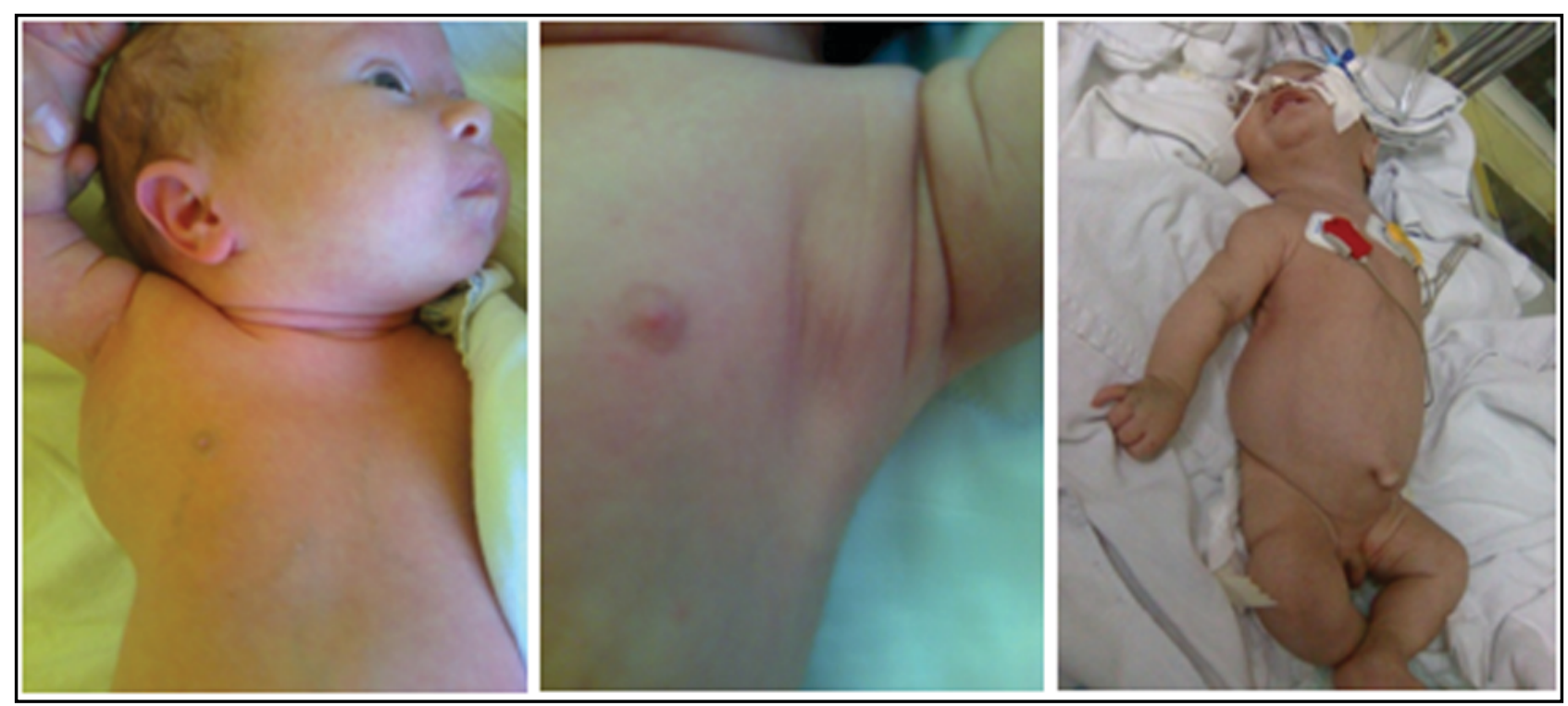

Figure 1 - Clinical aspects of a two-months-old infant: A. Pale skin, with multiple pemphigoid lesions; B. Axillary lymphadenopathy; C. Moderate abdominal distention, with mild umbilical hernia 
Clinical, laboratory and imaging findings suggested neonatal sepsis based on a X-linked immunodeficiency, chronic granulomatous disease (the infant is the only surviving male in this family, having 4 healthy sisters).

For complete diagnosis, oxidative burst assay was performed using Bursttest that found low oxidative activity of the neutrophils.

Differential diagnosis was made with other $\mathrm{X}$-linked immunodeficiency diseases with respiratory manifestations: Bruton's X-linked agamaglobulinemia and hyper IgM syndrome, excluded based on the normal level of immunoglobulins $\mathrm{IgG}$, IgA, mild elevated IgM.

The patient received large spectrum antibiotics, supportive transfusion therapy, i.v. immunoglobulins.

In evolution, fever persisted for a week, followed by progressive improvement in the next 20 days, with:

- Decreased adenopathy volume;

- No respiratory manifestations;

- Healing of the skin lesions;

- No ear secretion;

- One episode of acute enterocolitis, no germ

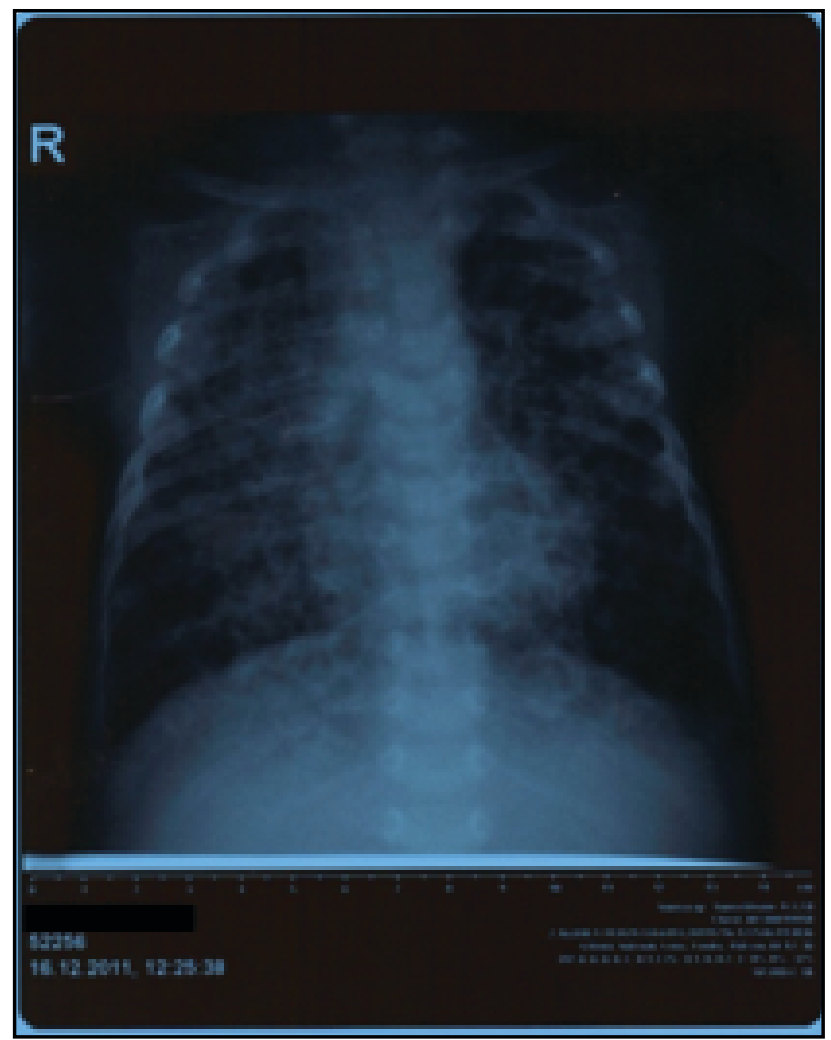

Figure 2 - Chest X-ray identified;

- Slow ponderal gain;

- Cholestasis syndrome, with transient jaundice, total bilirubin of $4 \mathrm{mg} / \mathrm{dl}$, direct bilirubin of $3.31 \mathrm{mg} / \mathrm{dl}$;

- Normal total blood count;

- Persistence of pulmonary radiological modifications. A computerized axial tomography scan of the chest revealed alveolar nodules with variable diameters $(5-10 \mathrm{~mm})$ in both pulmonary fields.

The patient is dismissed after 30 days of hospitalization, with prophylactic cotrimoxazole at home [2]. After 1month, is readmitted in the hospital and he deceases with sepsis, with the same first clinical aspect; the pulmonary disease produced respiratory insufficiency, unresponsive to the applied treatment.

Anatomopathological examination showed multiple microabscesses in the lungs and in the liver that confirmed the diagnosis.

Histological findings revealed multiple pulmonary granulomas, well defined, with round shape, containing transformed macrophages called epiteloid cells, along with lymphocytes, occasionally polimorphonuclear cells and fibroblasts [3].

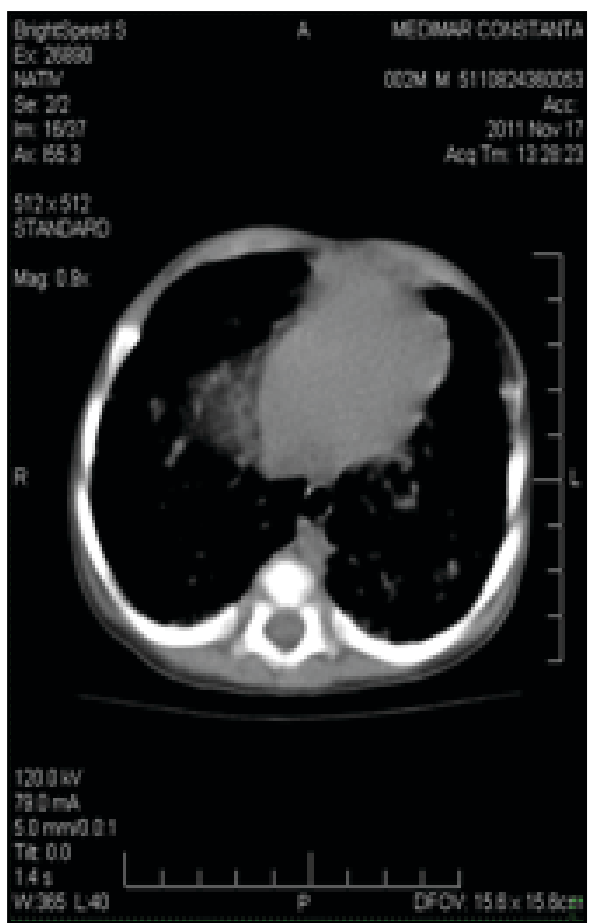

Figure 3 Chest CT scan 
CGD is caused by a defect in phagocytic NADPH oxidase, wich is responsabile for producing $\mathrm{O}^{2-}$. This superoxide anion is then converted to relatively bactericidal reactive oxidants, such as hydroxyl radical $(\mathrm{OH}-)$, hydrogen peroxide $(\mathrm{H} 2 \mathrm{O} 2)$, peroxinitrite anion (ONOO-) and oxihalides (HOX-, in which the $\mathrm{X}$ moiety is most commonly clorine). The superoxide anion is generated by transferring electrons from the reduced NADPH to molecular $\mathrm{O} 2$ in response to physiologic stimuli, such as phagocytosis. This reaction is mediated by the phagocyte NADPH oxidase otherwise known as phagocyte oxidase (phox)[1].

The most common molecular defect in CGD is a mutation in the CYBB (cytochrome $\mathrm{B}$, b subunit) gene that is located on the $\mathrm{X}$ chromosome and that encodes for glycoprotein 91(gp91). Gp91 deficiency accounts for $50-70 \%$ of all cases of CGD [4].

The CGD phagocyte can kill numerous microorganisms despite its defects because most microorganisms endogenously produce hydrogen peroxide, which the CGD-affected phagocyte can modify and use against the organism in the phagosome. Bacteria and fungi that cause most infections in CGD are catalase-positive organisms. These microorganisms produce catalase that breaks down endogenously produced hydrogen peroxide; the generation of oxygen radicals by a normally functioning phox system is needed to ensure the death of these infecting microorganisms [5].

The diagnosis of CGD should be considered in any patient with recurrent infections with catalasepositive organisms.

Bursttest is a flowcytometric test preferred for screening and diagnostic for CGD. Phagocytic cells reduce Dihydrorhodamine to the strongly fluorescent compound rhodamine. Individual fluorescent cells can be counted, and the amount of fluorescence per cell is quantified with flow cytometry. This test combines the best features of the slide and tube nitroblue tetrazolium tests [6].
The early onset of the symptoms is common for CGD. The suspicion of CGD should be based on clinical feature. The screening test for positive diagnosis is Bursttest.

CGD is characterized by recurrent infections and the prognosis is constantly the worst. Curative approaches are hematopoietic stem cell transplant and gene therapy in addition to medical therapy in acute or chronic infections [7].

\section{References}

1. LAWRENCE C. WOLFE, MD et al. - Pediatric Chronic Granulomatous Disease, 16 Apr 2010, [Medline]

2. SEGER RA. - Modern management of chronic granulomatous disease. $\mathrm{Br} \mathrm{J}$ Haematol. Feb 2008;140(3):255-66. [Medline].

3. DOHIL M, PRENDIVILLE JS, CRAWFORD RI, SPEERT DP. - Cutaneous manifestations of chronic granulomatous disease. A report of four cases and review of the literature. $\mathrm{J}$ Am Acad Dermatol. Jun 1997;36(6 Pt 1):899-907. [Medline].

4. BJORGVINSDOTTIR H, DING C, PECH N, et al. - Retroviral-mediated gene transfer of gp91phox into bone marrow cells rescues defect in host defense against Aspergillus fumigatus in murine X-linked chronic granulomatous disease. Blood. Jan 1 1997;89(1):41-8. [Medline].

5. BARTON LL, MOUSSA SL, VILLAR RG, HULETT RL. - Gastrointestinal complications of chronic granulomatous disease: case report and literature review. Clin Pediatr (Phila). Apr 1998;37(4):231-6. [Medline].

6. JESSICA S. KOSUT, MD; NAYNESH R. KAMANI, MD; BARBARA A. JANTAUSCH, 
MD. - One-Month-Old Infant With Multilobular Round Pneumonias. Pediatric Infect Dis J. 2006; 25(1):95,97 Lippincott Williams \&Wilkins

7. MIKI M, ONO A, AWAYA A, et al.- Successful bone marrow transplantation in chronic granulomatous disease. Pediatr Int. Dec 2009;51(6):838-41. [Medline]. 